\title{
Complex dynamics of financial indices
}

\section{J.A. Tenreiro Machado}

\begin{abstract}
This paper presents a novel method for the analysis of nonlinear financial and economic systems. The modeling approach integrates the classical con- cepts of state space representation and time series re- gression. The analytical and numerical scheme leads to a parameter space representation that constitutes a valid alternative to represent the dynamical behavior. The results reveal that business cycles can be clearly revealed, while the noise effects common in financial indices can elegantly be filtered out of the results.
\end{abstract}

Keywords Time series,Complex dynamics, Financial analysis, State space, Trendlines

\section{Introduction}

Financial indices play a fundamental role in mankind activities. The time series reveal complex dynamical phenomena and considerable efforts have been made to involve mathematical tools usual in the analysis of nonlinear dynamics $[2,18,26,27]$. In spite of the ef- forts that have been devoted to this topic, the true is that "randomness" plays still a fundamental role when working with these kind of objects $[3,9,20,22,25]$. This state of affairs somehow points toward adopting statistical or stochastic analytical methods and, consequently, precluding modeling perspectives closer to those common in electrical, mechanical, thermal, and other types of physical systems. This paper emerges from realizing that this classical paradigm for system modeling needs to be adapted to financial systems and proposes a new approach for overcoming some of the aforementioned limitations.

Financial signals reflect the dynamics of a complex system where the concepts of measure, variable, pa- rameter, and model are not clearly defined as occurs in physics or engineering. The "financial system," under- lying the index evolution, is composed by a multitude of different agents, exhibiting a plethora of phenom- ena with distinct nature and size that interplay both between themselves and the "economical system." Be- sides these difficulties of defining an assertive mod- eling paradigm, financial indices reveal a noisy be- haviour with chaotic characteristics. This fact poses numerical problems for calculating derivatives and, therefore, it is not straightforward adopting tools usual in dynamical systems such as the state space repre- sentation. Phase variables constitute a common choice for state variables, since they require the consecutive time derivatives and are a solid option for construct- ing trajectories representative of the system dynam- ics. Nevertheless, such option is avoided in the case of financial dynamics due to the heavy noise present in 
the indices. The noise filtering is often discharged a priori since a strong intervention perturbs not only noise, but also the signals that are under evaluation. 
Fig. 1 Time evolution of the Dow Jones Industrial Average and the Europe Brent Spot Price FOB, from 18 May 1987 up to 12 April 2013 (6,760 points)

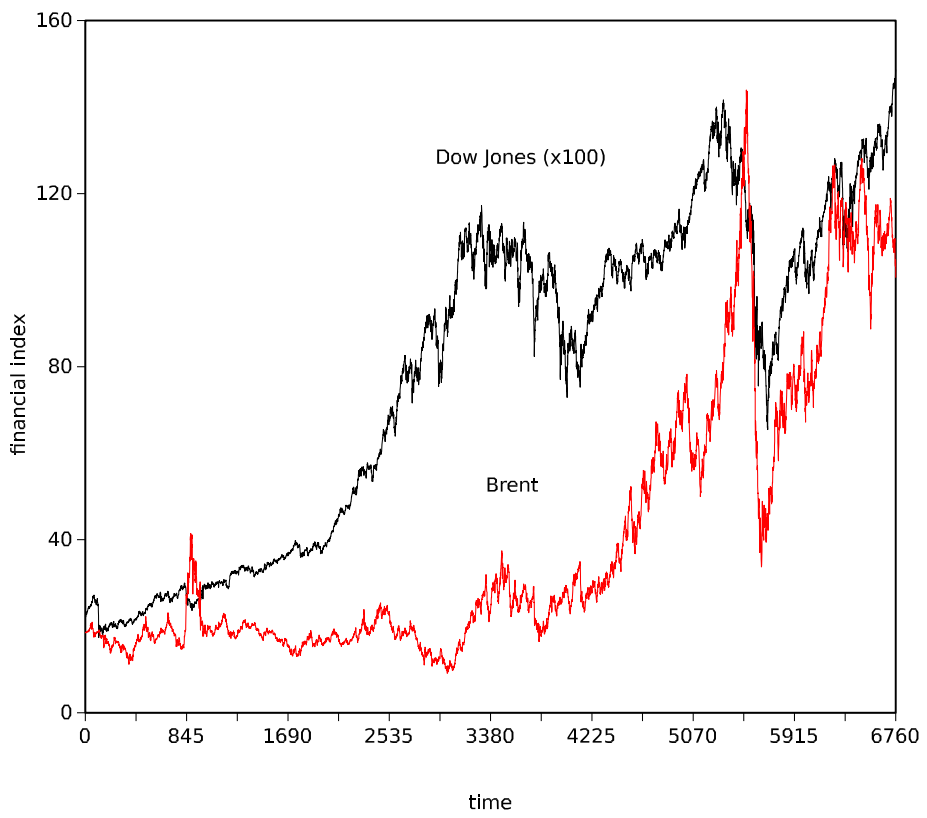

Several other options were proposed to overcome that problem, namely the use of pseudo state space [4], the adoption of fractional derivatives [8], the analy- sis using transforms $[11,15,17]$, the study by means of visualization tools $[12,13]$, the formulation of fil- tering as an inverse optimization problem [10], or de- scribing the dynamics in the viewpoint of power law regressions $[14,16]$. This paper adopts a new strat- egy by reformulating the problem of calculating the derivative. The newly proposed method discharges the noise and preserves the trend of the financial dynam- ics by considering regressions at several time scales.

The resulting parameters are then used for represent-

ing the dynamics in a multidimensional plot that be-

haves similarly to the classical phase space. Further-

more, this strategy leads to an algorithm establishing a compromise between time resolution and filtering, while leading to the direct analysis of the resulting plots.

Bearing these ideas in mind this paper is organized as follows. Section 2 formulates the new method, de- velops several experiments with two financial indices and discusses the results. Finally, Sect. 3 draws the main conclusions. gle and by obtaining its slope. Such slope represents the average over the interval. Consequently, reducing the interval length approximates the slope of the tri- angle up to the value of the derivative. Nevertheless, at small time scales, the effect of noise predominates. Often in financial analysis we are interested in the trend over a given time window and we simply look at the global signal evolution without paying atten- tion to small (noisy) phenomena. Following this line of thought, for a given signal $f(t)$, where $t$ denotes time, the proposed method calculates a trendline $g(t)$ that approximates $f(t)$ over an interval $t \in T$. The derivative now can be obtained from $\frac{d g}{}$. This is the standard method of $\stackrel{d t}{d t}$ numerical derivatives calculating

\section{Modeling approach and experiments}

In the standard procedure for calculating the derivative of a function, we start by constructing a trian- 
when adopting polynomials for $g(t)$ and a few points for $T[5,7,23]$. In the proposed algorithm, we shall preserve the initial idea of having a considerable num- ber of points in $T$ embedded with a scheme for defin- ing the appropriate time scale. Therefore, we divide

iteratively the domain of $f(t)$ into $2^{n-1}, n=1,2, \ldots$, time windows of identical size. Obviously, the higher the value of $n$, the smaller the number of points in the corresponding interval $T_{n}$ and the stronger the effect of noise upon the calculations. The choice for a par-ticular value of $n$, that is, the choice of a given time scale, is for the user to decide based upon a compro- mise between instantaneous behavior and noise limita- tion. The second aspect of the algorithm is the choice of $g(t)$. In the present case, we consider "robust" func- 
Fig. 2 Dependency tree in the locus $\{a, b\}$ for the Dow Jones Industrial Average, $n=4$ and $n=7$, linear trendline
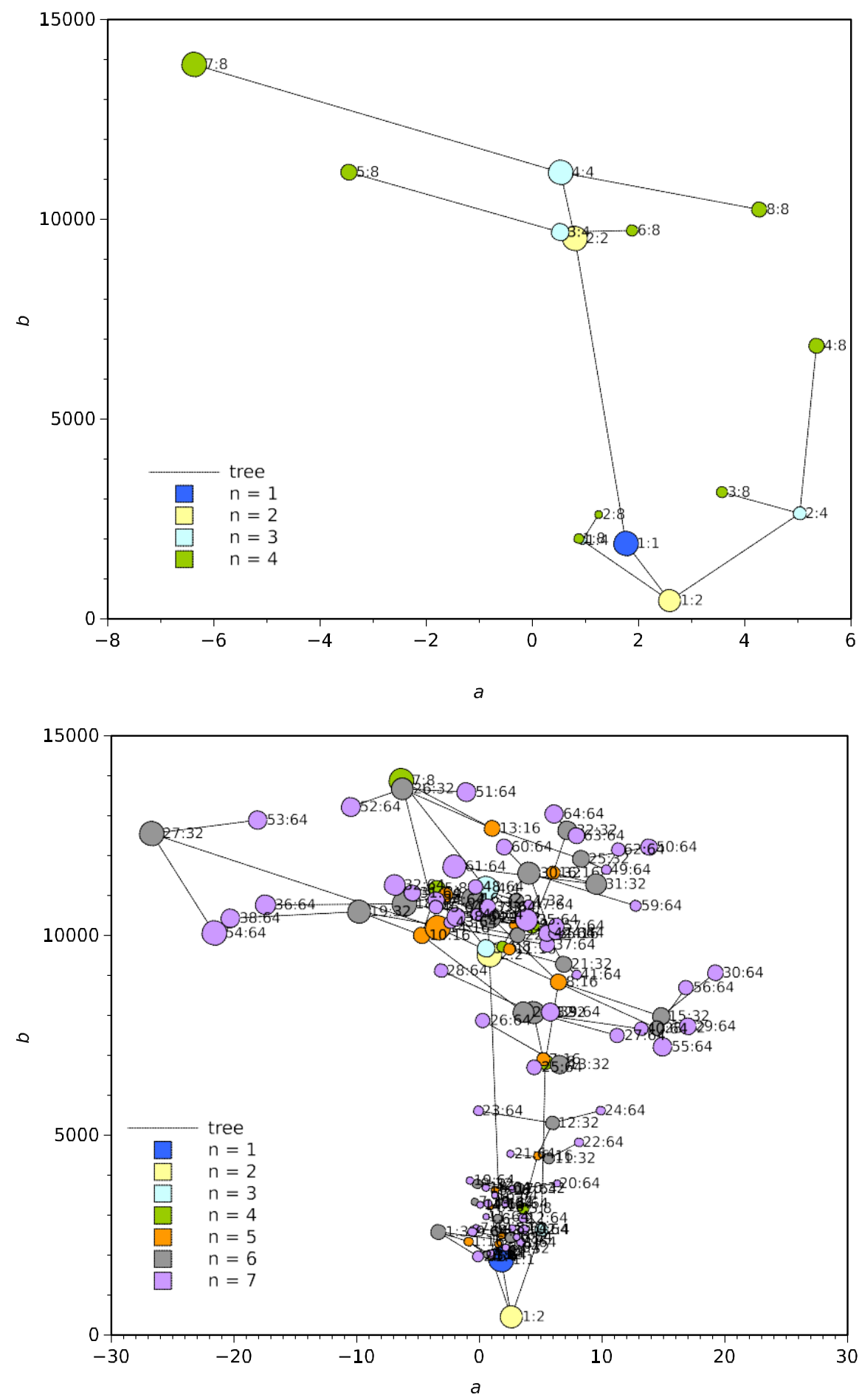
Fig. 3 Locus $\{a, b\}$ for the Dow Jones Industrial Average, $n=4$, and $n=7$, linear trendline
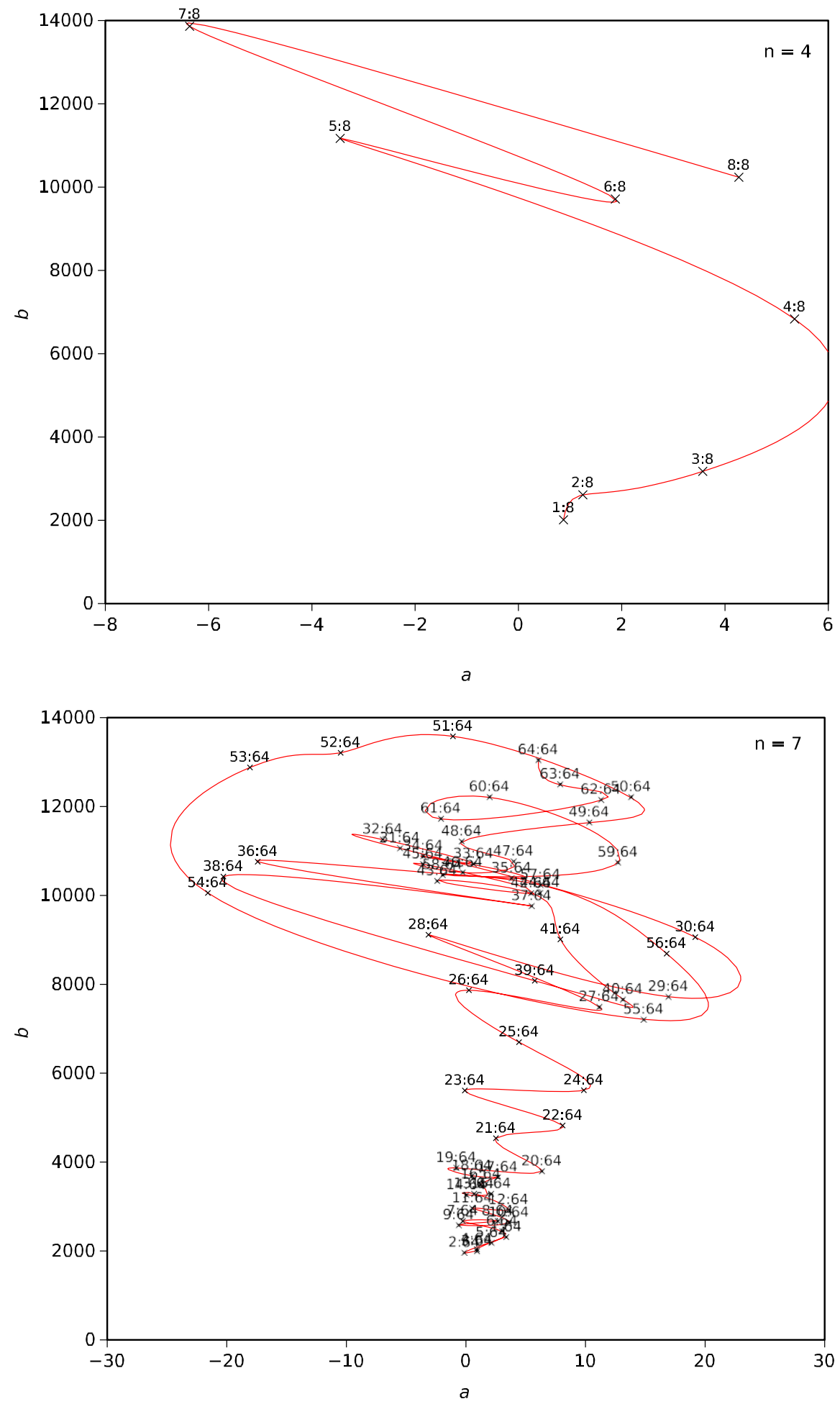
Fig. 4 Dependency tree in the locus $\{a, b\}$ for the Dow Jones Industrial Average, $n=5$, linear trendline

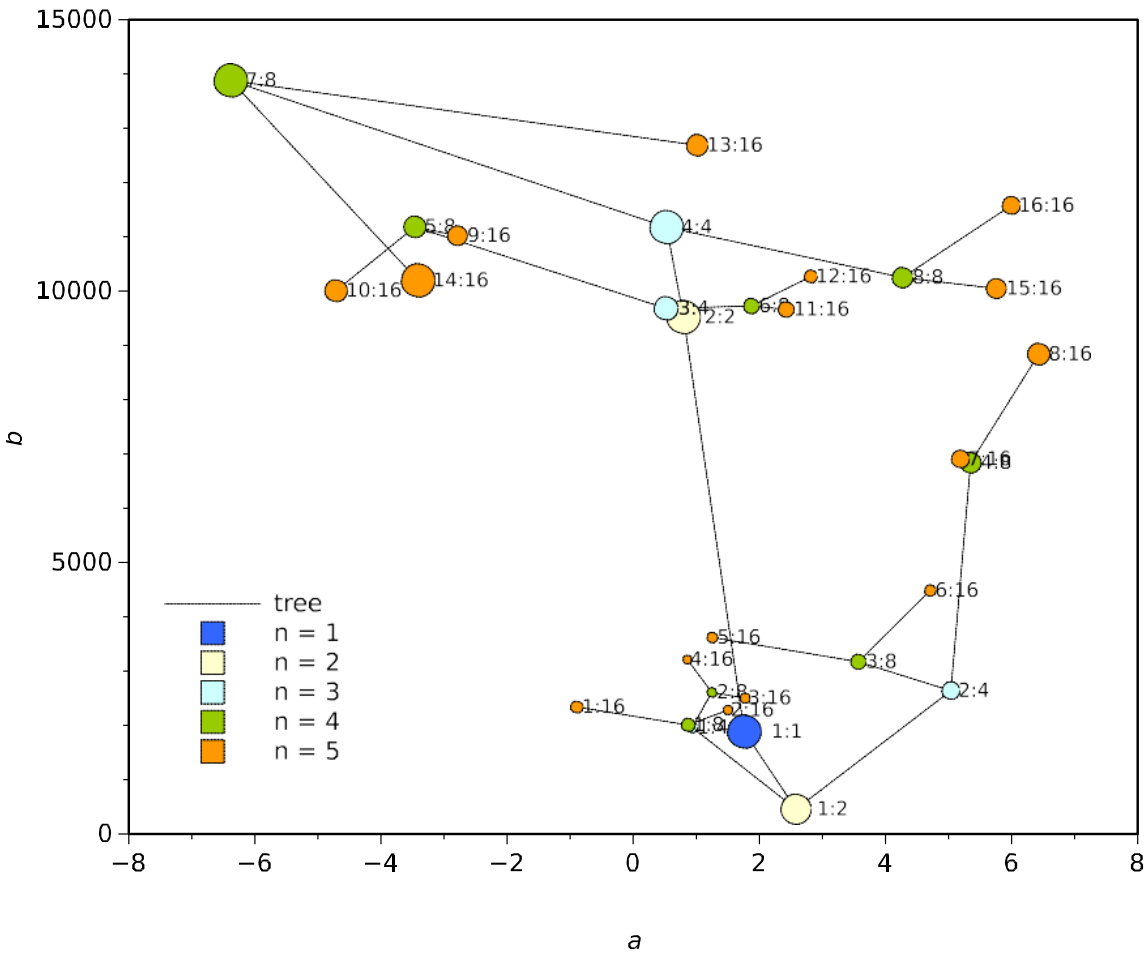

Figure 1 shows the time evolution of the Dow Jones Industrial Average and the Europe Brent Spot Price FOB over period $T_{1}$.

Figure 2 depicts the dependency tree in the locus $\{a, b\}$ for the Dow Jones Industrial Average, $n=4$ and $n=7$, linear trendline, in period $T_{1}$. The circles are proportional to the mean root mean square error over each time window and the labels $p: q$ mean the $p$ th time window for a total of $q$ intervals. The $a$-axis reflects the slope of the financial index and, therefore,

the first/second quadrant means a positive/negative cydays and holidays that lead to some lack of data in the original time series, were estimated by simple interpolation of the neighbor val- ues, so that all weeks include 5 days, making a total of 6,760 points.

We adopt numerical experiments for two financial indices, representing the Dow Jones Industrial Aver- age and the Europe Brent Spot Price FOB (in dollars per barrel) available at the websites of the "Yahoo! Fi- nance" and the "US Energy Information Administra- tion," respectively. The time series include daily val- ues in period $T_{1}$ starting at 18 May 1987 and ending at 12 April 2013. Special 
cle. On the other hand, the $b$-axis reflects the value of the index itself. It is clear that the larger the number of intervals $n$, the more intricate the tree becomes and the larger the dispersion particularly for $a$. Another as- pect that deserves to be highlighted is the choice of a succession of time bisections. That algorithm would lead to the requirement of a total number of points performing a power of two. To alleviate this restriction, during the time scale formulation, several limit points are not considered so that each one has an even num- ber of points. Since the number of points remaining in each interval is still large, the effect of such truncation

upon the result is negligible. Figure 3 shows the parameter locus $\{a, b\}$ (i.e., a mimic of the phase plane). The dots represent the estimated parameters $a_{k}$ and $b_{k}$, 

Fig. 5 Locus $\{a, b\}, n=5$, of the Dow Jones Industrial Average, for linear and exponential trendlines
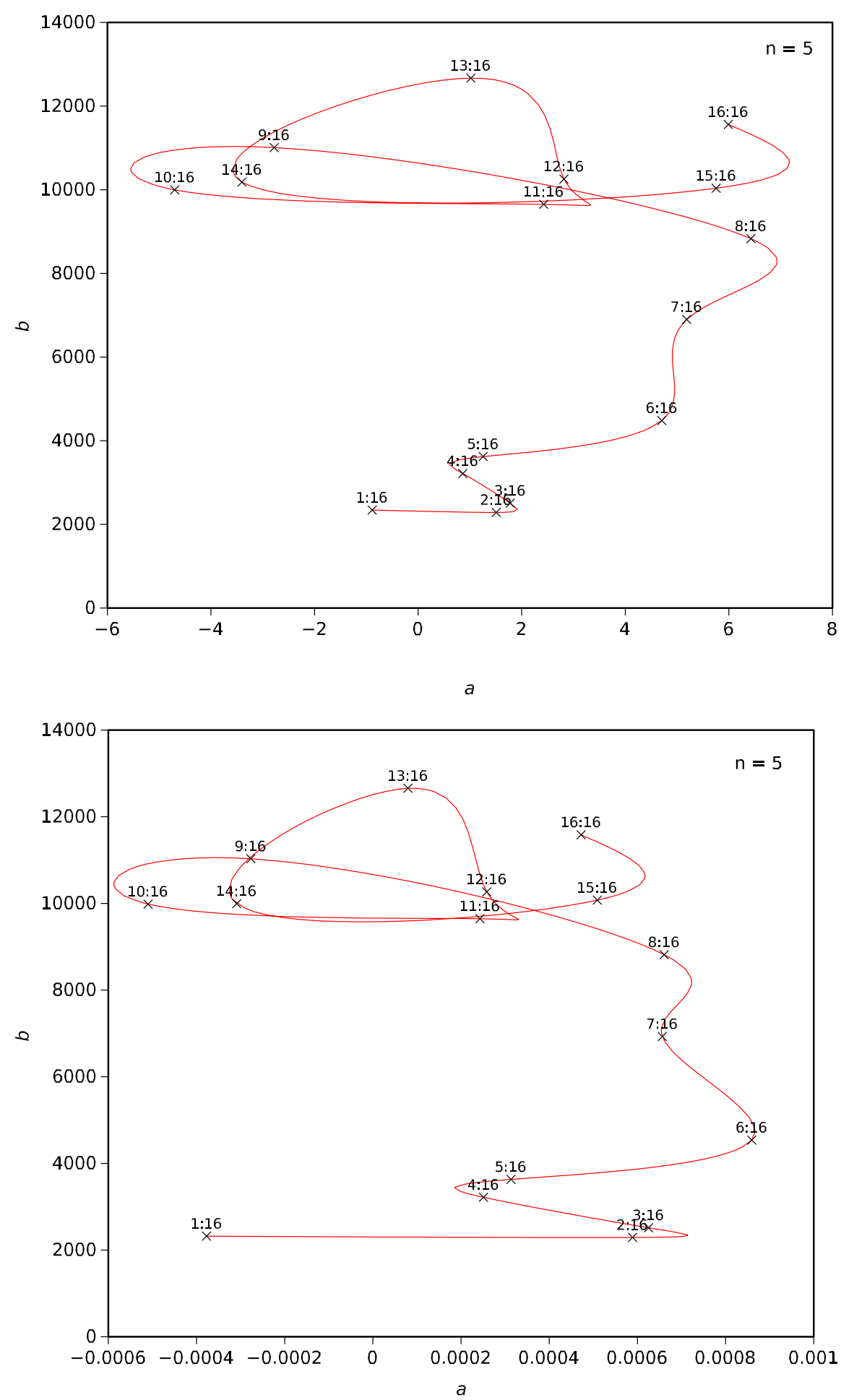
Fig. 6 Locus $\{a, b\}, n=5$, of the Europe Brent Spot Price $F O B$, for linear and exponential trendlines
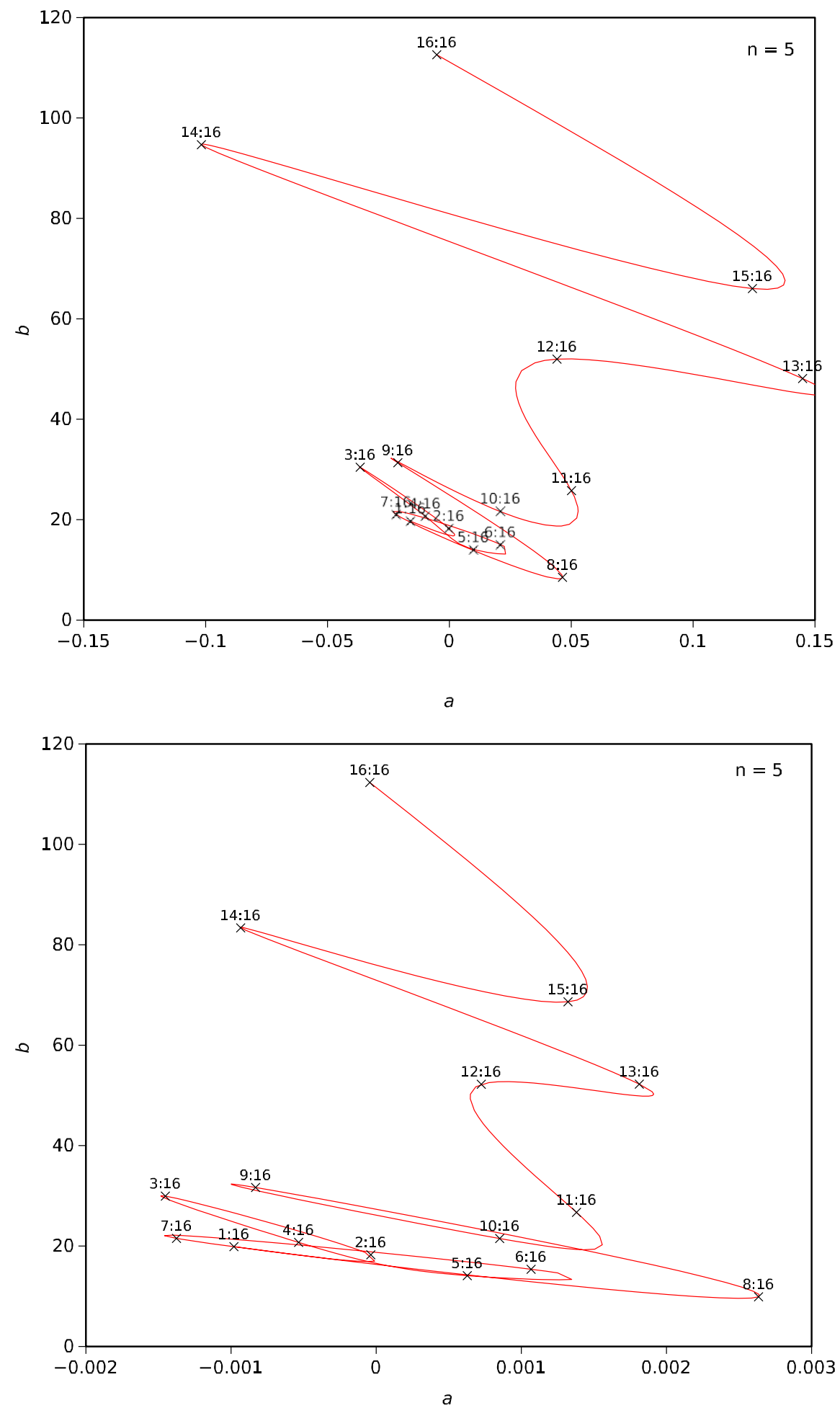
Fig. 7 Locus $\{a, b, c\}, n$ $=5$, of the Dow Jones Industrial Average, for parabolic trendline

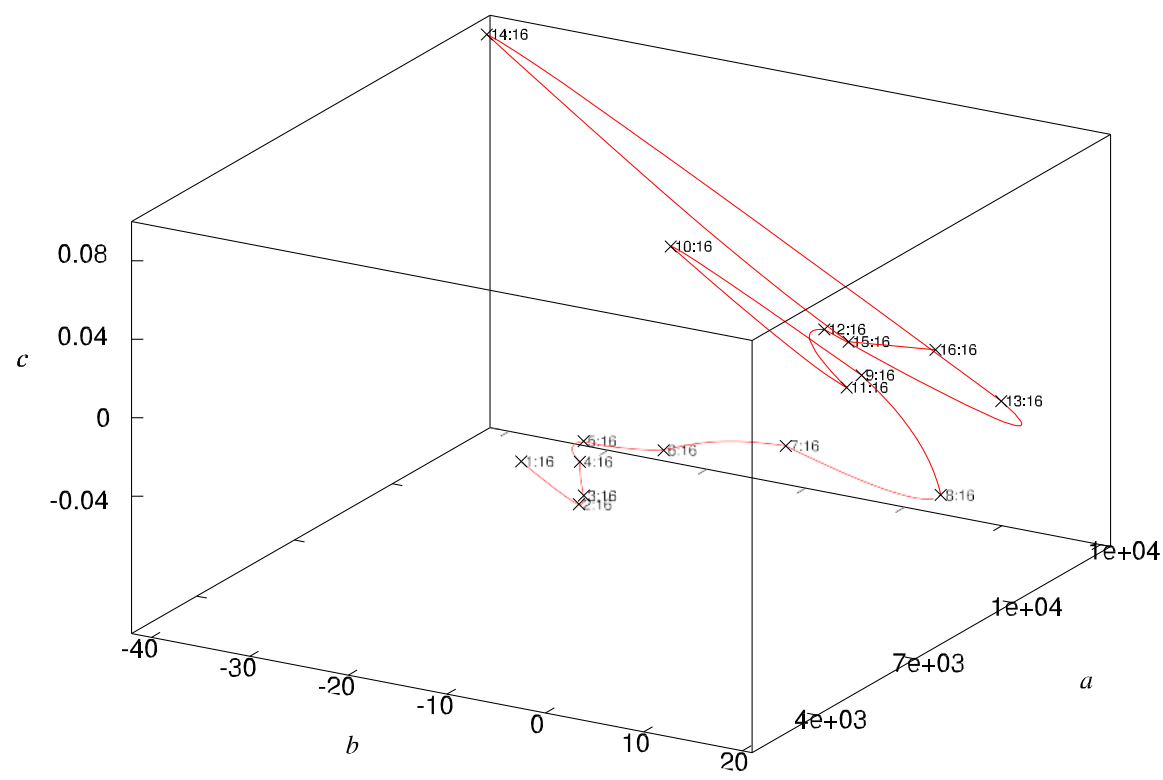

$\{13: 16\} \rightarrow\{14: 16\} \rightarrow\{15: 16\} \rightarrow\{16: 16\}$. This $k=1, \ldots, n$ for each period and the connecting trajectories. We observe that $n=3$ leads to an oversimplification, while the case $n=7$ reflects the noisy behavior of the original time series.

Several experiments demonstrated that $n=5$ establishes a good compromise between precision and noise. For example, Fig. 4 depicts the dependency tree

in the locus $\{a, b\}$ for the Dow Jones Industrial Average, $n=5$, and linear trendline. Figures 5 and 6 show the locus $\{a, b\}, n=5$, for the Dow Jones Industrial Average and the Europe Brent Spot Price FOB, respectively, when adopting the linear and exponential trendlines.

We should note that the meaning of parameters $a$ and $b$ vary with the trendline $g$. Nevertheless, the two plots are of the same type and lead to identical con- clusions. This means also that there is no special rea- son for selecting one particular type of trendline. In what concerns the dynamics of the Dow Jones In- dustrial Average, we observe four phases: a first in-

creasing trajectory $\{1: 16\} \rightarrow\{8: 16\}$, two repetitive cycles $\{8: 16\} \rightarrow\{11: 16\}$ and $\{11: 16\} \rightarrow\{15:$ $16\}$, and finally a (still) indeterminate trajectory for

$\{15: 16\} \rightarrow\{16: 16\}$ that presently is in a positive state. For the Europe Brent Spot Price FOB, we observe a distinct behavior, namely three small initial cy- cles for $\{1: 16\} \rightarrow\{9: 16\}$, an increasing trajectory for $\{9: 16\} \rightarrow\{13: 16\}$, and an oscillatory behavior, composed of alternative and positive trajectories, for 
behavior can be recognized directly at the time evolu- tion represented in Fig. 1, but as occurs in state space representations, we obtain a much clear picture of the overall dynamics.

In the analysis of dynamical systems, often is required the adoption of more than two state variables. However, the use of a second-order derivative in the present time series is clearly a problematic option. Therefore, it is relevant to investigate if the proposed method can be generalized for a larger number of di- mensions. In this line of thought, we consider a 3dimensional representation supported by the parabolic

and Harris trendlines $g(t)=a_{k}+b_{k} t+c_{k} t^{2}$ and $g(t)$ $=\left(a_{k}+b_{k} t^{c_{k}}\right)^{-1}, a_{k}, b_{k}, c_{k} \in R, k=1, \ldots, n$, respectively. Both trendlines involve three parameters

reflecting distinct "dynamical properties" and, there- fore, making them suitable for a threedimensional representation. Nevertheless, it is possible to adopt other types of functions revealing better/worst proper- ties for each specific type of time series. In particular, the parameter $c$ required by the Harris model, reflects the power law behavior known in fractional order dy- namics [1, $6,19,21,24]$.

Figures 7 and 8 depict the locus $\{a, b, c\}, n=5$, for the Dow Jones Industrial Average when adopting the

parabolic and Harris regressions.

The Harris model seems to be slightly superior to the parabolic regression because we verify that the third dimension is useful is discriminating the complex dynamics that appears in the final period of time. Fur- 
Fig. 8 Locus $\{a, b, c\}, n$ $=5$, of the Dow Jones Industrial Average, for Harris trendline

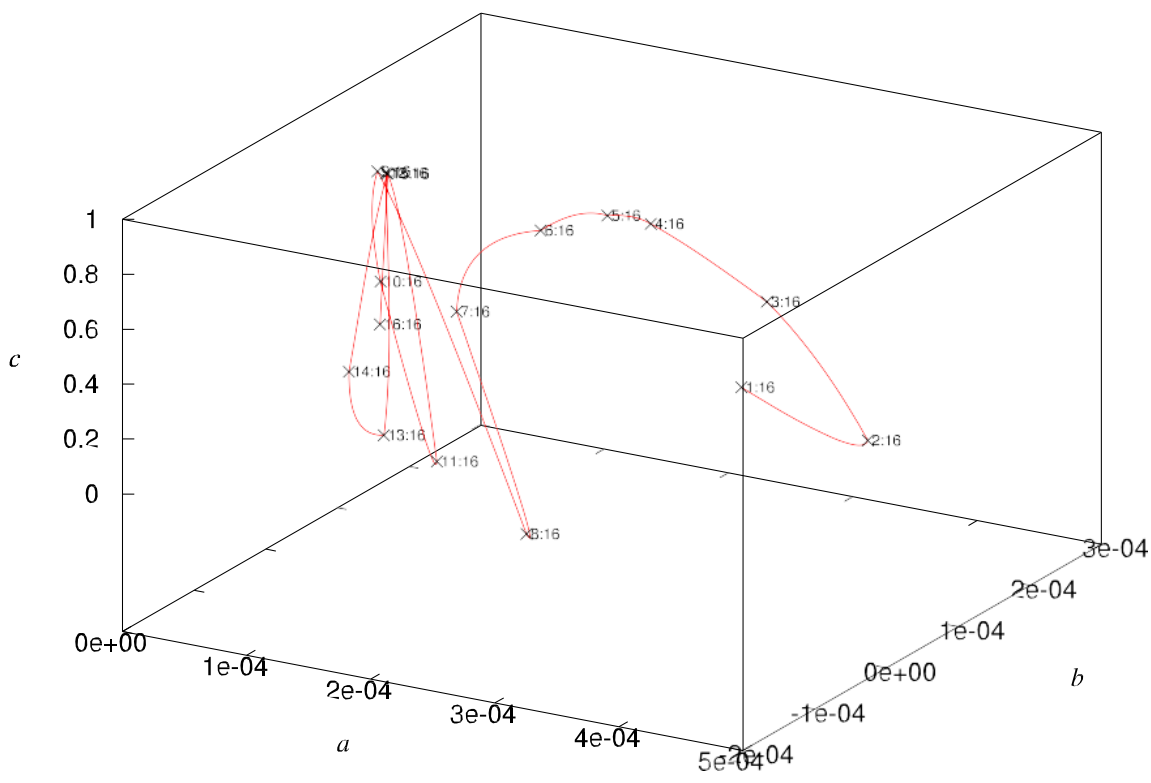

thermore, we observe a smooth evolution at the first part and two large loops at the final part of the trajec- tory. The large loops demonstrate a strong dynamical instability and a kind of strange attractor influencing present day financial dynamics. These results are in ac- cordance with those depicted by the two-dimensional charts, but provide a better visualization.

In conclusion, we verified that the parameter space constitutes a valid alternative to the classical state space representation, namely by avoiding noise effects that are present in financial time series.

\section{Conclusions}

This study addressed the analysis of complex and nonlinear dynamics in financial systems. Markets are characterized by means of indices with considerable noise making difficult the application of state space representations. The proposed methodology reformu- lates the classical methods leading to a new model based in the trajectory evolution in the parameter space. Financial cycles and crises are clearly visible since noise effects are eliminated.

1. Baleanu, D., Diethelm, K., Scalas, E., Trujillo, J.: Fractional Calculus: Models and Numerical Methods. World Scientific, Boston (2012)

\section{References}


2. Bischi, G.I., Chiarella, C., Gardini, L. (eds.): Nonlinear Dynamics in Economics, Finance and the Social Sciences. Springer, Berlin (2010)

3. Chen, W.C.: Nonlinear dynamics and chaos in a fractional- order financial system. Chaos Solitons Fractals 36, 1305- 1314 (2008)

4. Duarte, F.B., Machado, J.A.T., Duarte, G.M.: Dynamics of the Dow Jones and the NASDAQ stock indexes. Nonlinear Dyn. 61(4), 691-705 (2010)

5. Khan, I.R., Ohba, R.: New finite difference formulas for numerical differentiation. J. Comput. Appl. Math. 126(1-2), 269-276 (2000)

6. Kilbas, A., Srivastava, H., Trujillo, J.: Theory and Applications of Fractional Differential Equations. NorthHolland Mathematics Studies, vol. 204. Elsevier, Amster- dam (2006)

7. Li, J.: General explicit difference formulas for numerical differentiation. J. Comput. Appl. Math. 183(1), 2952 (2005)

8. Lin, G., Feeny, B.F., Das, T.: Fractional derivative reconstruction of forced oscillators. Nonlinear Dyn. 55(3), 239- 250 (2009)

9. Ma, J., Bangura, H.I.: Complexity analysis research of financial and economic system under the condition of three parameters' change circumstances. Nonlinear Dyn. 70(4), 2313-2326 (2012)

10. Machado, J.A.T.: Calculation of fractional derivatives of noisy data with genetic algorithms. Nonlinear Dyn. 57(1-2), 253-260 (2009)

11. Machado, J.A.T., Duarte, G.M., Duarte, F.B.: Analysis of financial data series using fractional Fourier transform and multidimensional scaling. Nonlinear Dyn. 65(3), 235-245 (2011)

12. Machado, J.A.T., Duarte, G.M., Duarte, F.B.: Identifying economic periods and crisis with the multidimensional scaling. Nonlinear Dyn. 63(4), 611-622 (2011) 
13. Machado, J.T., Duarte, F.B., Duarte, G.M.: Analysis of stock market indices through multidimensional scaling. Commun. Nonlinear Sci. Numer. Simul. 16(12), 46104618 (2011)

14. Machado, J.A.T., Duarte, G.M., Duarte, F.B.: Fractional dynamics in financial indexes. Int. J. Bifurc. Chaos Appl. Sci. Eng. 22(10), 1250249 (2012)

15. Machado, J.T., Duarte, F.B., Duarte, G.M.: Analysis of financial indices by means of the windowed Fourier transform. Signal Image Video Process. 6(3), 487-494 (2012)

16. Machado, J.T., Duarte, F.B., Duarte, G.M.: Power law analysis of financial index dynamics. Discrete Dyn. Nat. Soc. 2012, 120518 (2012)

17. Machado, J.T., Duarte, G.M., Duarte, F.B.: Analysis of stock market indices with multidimensional scaling and wavelets. Math. Probl. Eng. 2012, 819503 (2012)

18. Meyers, R.A. (ed.): Complex Systems in Finance and Econometrics. Springer, New York (2010)

19. Miller, K., Ross, B.: An Introduction to the Fractional Calculus and Fractional Differential Equations. Wiley, New York (1993)

20. Ngounda, E., Patidar, K.C., Pindza, E.: Contour integral method for European options with jumps. Commun. Nonlinear Sci. Numer. Simul. 18(3), 478-492 (2013)
21. Oldham, K., Spanier, J.: The Fractional Calculus: Theory and Application of Differentiation and Integration to Arbitrary Order. Academic Press, New York (1974)

22. Piqueira, J.R.C., Mortoza, L.P.D.: Complexity analysis research of financial and economic system under the condi- tion of three parameters' change circumstances. Commun. Nonlinear Sci. Numer. Simul. 17(4), 1690-1695 (2012)

23. Qu, R.: A new approach to numerical differentiation and integration. Math. Comput. Model. 24(10), 55-68(1996)

24. Samko, S., Kilbas, A., Marichev, O.: Fractional Inte- grals and Derivatives: Theory and Applications. Gordon \& Breach, Amsterdam (1993)

25. Silva, S.D., Matsushita, R., Gleria, I., Figueiredo, A., Rathie, P.: International finance, Lévy distributions, and the econophysics of exchange rates. Commun. Nonlinear Sci. Numer. Simul. 10(4), 355-466 (2005)

26. Trippi, R.: Chaos \& Nonlinear Dynamics in the Financial Markets. Irwin, Burr Ridge (1995)

27. Vialar, T.: Complex and Chaotic Nonlinear Dynamics. Advances in Economics and Finance, Mathematics and Statistics. Springer, Berlin (2009) 